Proceedings

\title{
Covid-19 Pandemic in Brazil: Clinical manifestation and effect of comorbidities on outcomes of hospitalized SARI cases ${ }^{\dagger}$
}

\author{
Mario Keko* and Karl E. Peace
}

Department of Biostatistics, Epidemiology and Environmental Sciences, Jiann Ping-Hsu College of Public Health, Georgia Southern University, 501 Forest Drive, Statesboro 30458, GA, USA; peacekarl@frontier.com

* Correspondence: mk12625@georgiasouthern.edu; Tel.: +19122433283.

Publisher's Note: MDPI stays neutral with regard to jurisdictional claims in published maps and institutional affiliations.

\section{(c) (i)}

Copyright: (c) 2021 by the authors. Submitted for possible open access publication under the terms and conditions of the Creative Commons Attribution (CC BY) license (http://creativecommons.org/licenses/by/4.0/).

\begin{abstract}
Hospitalized SARI cases of 2020 reported to the Ministry of Health of Brazil through the SIVEP Gripe system are subject to our analysis. They are classified as Covid and non-Covid and clinical manifestations and comorbidities are reported for each group. The time trend in the number of cases reported in 2020 is compared to the previous year and the performance of the PCR test is explored in each group. The proportion of death is reported among different subgroups of the patients by epidemiological week. Logistic and Poisson regression models are used to check the effect of comorbidities on clinical outcomes.
\end{abstract}

Keywords: Covid-19; Clinical manifestation; Outcomes; Comorbidities

\section{Introduction}

Covid-19 refers to the Severe Acute Respiratory Syndrome Coronavirus 2 (SARSCoV-2) which causes Severe Acute Respiratory Infection [1]. It emerged in Wuhan, China in early December 2019 and later was classified as pandemic by the World Health Organization [1]. Its most common clinical manifestations include fever, cough, dyspnea, expectoration, headache, and myalgia or fatigue while diarrhea, hemoptysis, and shortness of breath are less common [2]. Research shows that the presence of comorbidities, like high blood pressure and diabetes is associated with a worse prognosis, especially in older patients [2]. Molecular diagnostic technology is crucial for the control of the virus spread [3], and it is accompanied by great challenges as well as opportunities for fast adaptations [4].

Brazil is one of the hardest-hit countries and is considered a global hotspot for the virus [5]. In this research work, we are presenting findings from the analysis of hospitalized Severe Acute respiratory Infection (SARI) cases in Brazil focused on getting a view of the clinical manifestations, outcomes like admission to ICU, use of non Invasive Ventilatory Support (non-IVS) and Invasive Ventilatory Support (IVS), length of hospitalization and ICU stay, and the effect of comorbidities on outcomes. We are also interested in whether there is an improvement over time in the proportion of SARI patients who are cured. Lastly, we have investigated the time trend of reported SARI cases and explored how it can be related to criteria for case classification and the role of PCR test performance.

\section{Materials and Methods}

\subsection{Data Source and Preparation}

The data used for this analysis are publicly available from IVIS Platform (Plataforma Integrada de Vigilância em Saúde) under the Ministry of Health of Brazil and are lastly updated on 13th October 2020 [6]. More specifically, it consists of hospitalized SARI patients reported in SIVEP Gripe (Sistema de Informação de Vigilância Epidemiológica da Gripe) surveillance system which is used to report individuals of any age hospitalized with [fever and (cough or throat pain)] and who have [dyspnea or $\mathrm{O}_{2}$ blood saturation 
$<95 \%$ or respiratory discomfort) [7]. Also, there are reported cases of death caused by SARI independent of hospitalization status [7]. For the purposes of our analysis, there is used the dataset of SARI cases reported in 2020 and it is the main focus; in addition, the 2019 dataset is used to compare the time trends of cases.

The dataset of the year 2020 was downloaded and observations for whom the final classification of the etiologic agent was missing and those who had biologically nonplausible observations were deleted first. In the end, there were 617,020 observations for analysis. Based on whether SARS-Cov-2 was the etiologic agent in the final case classification, two groups were created: Covid and non-Covid (etiologic agent different from SARS-Cov2). Taking into consideration the fact that the record sheet instructs reporting the presence of any specific comorbidity with X [7], it was assumed that where nothing was specified, the comorbidity was not present. Duration of hospitalization (and ICU stay) was calculated as the difference in days between the reported date of start and end of hospitalization (ICU stay). The classification of severity was based on signs and symptoms following National Institutes of Health (NIH) [8], (Table A1 in Appendix A). Results of PCR test or other tests of molecular biology were reported in one single variable and here are referred to as PCR.

\subsection{Methods}

Data were imported in SAS/STAT software, version 9.4, and appropriate procedures were used to generate descriptive analysis results, including proportions, means, and crosstabulations. R software, version 4.0.3 was used to generate appropriate graphs.

Full logistic regression models were used to estimate the effect predictors on ICU admission, type of Ventilatory Support (VS), and final case evolution following the methodology of Agresti [9]. The methodology of the same author was implemented to run full Poisson regression models to estimate the effect of predictors on length of hospitalization and ICU stay [10].

\section{Results}

\subsection{Demographic Characteristics of the Patients}

Table 1. Demographic characteristics.

\begin{tabular}{|c|c|c|c|c|}
\hline Variable & Categories & Covid (\%) & non-Covid(\%) & \\
\hline \multirow{3}{*}{ Sex } & Male & $206,836(56.7)$ & $132,783(52.7)$ & Of 617,020 observations, 364,904 \\
\hline & Female & $157,984(43.3)$ & $119,219(47.3)$ & Covid cases. Table 1 \\
\hline & Missing & 84 & 114 & 1 \\
\hline \multirow{4}{*}{ Race } & White & $17,771(6.5)$ & $13,024(6.6)$ & the distributions of \\
\hline & Black & $124,863(45.8)$ & $98,909(50.3)$ & sex, race, and education in both \\
\hline & Other & $129,858(47.7)$ & $84,559(43.0)$ & groups are comparable; also, ed- \\
\hline & Missing & 92,412 & 55,624 & reat \\
\hline \multirow{6}{*}{ Education } & No educatic & $10,194(8.1)$ & $10,203(12.1)$ & proportion of \\
\hline & $1^{\text {st }}$ cycle & $35,244(28.0)$ & $28,773(34.0)$ & the \\
\hline & $2^{\text {nd }}$ cycle & $23,557(18.7)$ & $15,649(18.5)$ & mean age is 52.8 in non-Covid \\
\hline & High school & $39,030(31.0)$ & $21,615(25.6)$ & cases and 59.7 in Covid cases. \\
\hline & University & $18,111(14.36)$ & $8,335(9.86)$ & \\
\hline & Missing & 238,768 & 167,541 & \\
\hline
\end{tabular}

\subsection{Clinical Manifestations and Outcomes by Group}

Among the reported signs and symptoms in Covid and non-Covid groups, cough $(80.6 \%$ and $74.2 \%)$, dyspnea $(79.8 \%$ and $77.3 \%)$, fever $(73.8 \%$ and $62.3 \%)$, respiratory discomfort $(70.0 \%$ and $69.9 \%), \mathrm{O}_{2}$ blood saturation $<95 \%(69.3 \%$ and $63.5 \%)$, and abnormal Chest X-Ray readings $(93.8 \%$ and $85.6 \%)$ are the most common. Throat Pain $(25.0 \%$ and $20.6 \%)$, diarrhea (18.3\% and $13.2 \%)$, and vomiting (11.1\% and $13.2 \%)$ are less common. 
When considering the three levels of severity (asymptomatic, mild, and severe), among the Covid group the frequency is $0.05 \%, 8.22 \%$, and $91.73 \%$. Among non-Covid patients, respective frequencies are $0.14 \%, 9.02 \%$, and $90.8 \%$ in those patients for whom severity could be determined from the reported signs and symptoms.

Among patients for whom the case evolution is known, the proportion of death is higher in Covid (40.0\% vs. $25.2 \%$ ). This group has a higher proportion of ICU admission ( $36.6 \%$ vs. $29.8 \%$ ), use of non-IVS ( $50.4 \%$ vs. $46.4 \%$ ), and use of IVS ( $21.2 \%$ vs. $16.8 \%)$. Considering case evolution by the epidemiological week in different subsets of the patients (Fig A1 in appendix), it can be observed that death proportion is consistently higher in Covid across all the subsets. In this group, the proportion of death is as high as $40 \%$ for all of them, 35\% in those receiving non-IVS, $60 \%$ in those admitted to ICU, and $75-80 \%$ in those receiving IVS. Furthermore, the mean of hospitalization length (10.8 vs. 8.6 days) and ICU stay (10.2 vs. 7.3 days) is higher in the Covid group. Fig A1 (a) in appendix A shows that the distribution of length of hospitalization and ICU stay is similar to a Poisson distribution; furthermore, the distribution in the Covid group is more shifted to the right.

\subsection{Time Trend of SARI Cases and the Performance of PCR Tests}

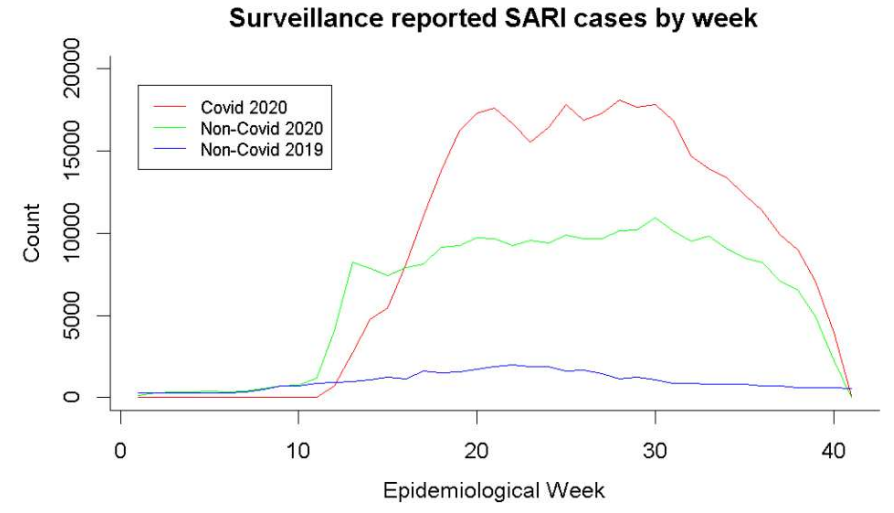

Figure 1. SARI cases reported on SIVEP Gripe by epidemiological week.

Table 2. Crosstabulation of confirmation criteria and the PCR test results.

\begin{tabular}{ccccccc}
\hline & Criteria & Detectable & $\begin{array}{c}\text { Not detect/in- } \\
\text { concl. }\end{array}$ & Waiting & Missing & Total \\
\hline \multirow{6}{*}{ Covid } & Lab. & $\mathbf{2 8 2 , 2 0 6 ( 8 3 . 1 )}$ & $8,731(2.6)$ & $17,593(5.2)$ & $31,040(9.1)$ & $339,570(93.1)$ \\
& E. Linking & $178(7.8)$ & $272(11.8)$ & $651(28.3)$ & $1,196(52.1)$ & $2,297(0.6)$ \\
& Clinical & $407(5.5)$ & $1,004(13.6)$ & $1,596(21.6)$ & $4,398(59.4)$ & $7,405(2.0)$ \\
& Other & $162(2.3)$ & $3,576(51.6)$ & $1,004(14.5)$ & $2,185(31.5)$ & $6,927(1.9)$ \\
& Missing & $4,474(51.4)$ & $432(5.0)$ & $1,868(21.5)$ & $1,931(22.2)$ & $8,705(2.4)$ \\
\hline \multirow{4}{*}{ Non- } & Lab & $4,771(2.2)$ & $\mathbf{2 0 3 , 6 4 0 ( 9 1 . 9 )}$ & $5,721(2.6)$ & $7,404(3.3)$ & $221,536(87.9)$ \\
Covid & E. Linking & $0(0.0)$ & $498(20.9)$ & $332(13.9)$ & $1,553(65.2)$ & $2,383(0.9)$ \\
& Clinical & $13(0.1)$ & $2,432(13.6)$ & $2,025(11.3)$ & $13,452(75.1)$ & $17,922(7.1)$ \\
& Other & $0(0.0)$ & $354(50.4)$ & $77(11.0)$ & $271(38.6)$ & $702(0.3)$ \\
& Missing & $105(1.1)$ & $6,082(63.5)$ & $1,424(14.9)$ & $1,962(20.5)$ & $9,573(3.8)$ \\
\hline
\end{tabular}

Non-Covid case reporting has increased (Fig 1) compared to last year, raising the question of whether there is a real increase or misdiagnosis. Table 1 indicates that among non-Covid patients on whom laboratory data are used for case confirmation, the proportion of PCR test being nondetectable/inconclusive is high (91.9\%) which is in contrast with the other group $(2.6 \%)$. Guidelines for case classification prioritize the laboratory results while clinical and epidemiological linking (E. linking) criteria should be used when those are inconclusive/not available [11]. There are other tests not using molecular technology that can be used for case classification, but PCR result being nondetectable/inconclusive 
in non-Covid suggests the presence of misdiagnosis. Fig A1 (b) shows no noticeable changes in the results of PCR tests by epidemiological week and also a huge contrast in PCR test results between the groups.

Trying to explore the factors that may be affecting the result of PCR test, we ran a logistic regression model with education as a predictor, while adjusting for age and sex. The odds of having a nondetectable/inconclusive result vs. detectable are 2.89 times higher $[2.77,3.01]$ in no education category, 2.32 times higher $[2.24,2.40]$ in fundamental $1^{\text {st }}$ cycle, 1.67 times higher $[1.61,1.73]$ in fundamental $2^{\text {nd }}$ cycle, and 1.25 times higher $[1.21,1.29]$ in high school as compared to university (reference). Similar effect sizes were observed when repeating the same model including race too. It is worth noting that for a considerable proportion of patients the education is missing. However, findings are consistent with another analysis applying a different methodology to an earlier data release and showing a positive relationship between higher income and Covid diagnosis [11].

While it has been stated that molecular diagnostic technology has been considered crucial in the prevention of the virus [3], we were also interested in the effect it could have on the case evolution. We ran a logistic regression model to estimate the effect of PCR result on dying from SARI while adjusting for the effect of age, sex, and severity. In the Covid group, the odds of dying were $5 \%$ lower $(\mathrm{CI}[-9 \%,-1 \%])$ in nondetectable/inconclusive and $5 \%$ higher in waiting (CI $[1 \%, 8 \%])$ as compared to the reference category of detectable result. Meanwhile, in the non-Covid group, the odds of death were $28 \%$ higher (CI [16\%, 41\%]) in nondetectable/inconclusive and 65\% higher (CI [47\%, 85\%]) in waiting as compared to PCR test being detectable. This suggests that the patients who have nondetectable/inconclusive and waiting PCR results and are classified as non-Covid have increased odds of death.

\subsection{Comorbidities and Their Effect on Outcomes}

Table 3. Effect of comorbidities, VS, age, race, and sex on outcome variables.

\begin{tabular}{|c|c|c|c|c|c|c|c|c|}
\hline & \multicolumn{2}{|c|}{ ICU admission } & \multicolumn{2}{|c|}{ Non-IVS vs. no VS } & \multicolumn{2}{|c|}{ IVS vs. no VS } & \multicolumn{2}{|c|}{ Dead vs. cured } \\
\hline & $\beta$ & OR & $\beta$ & OR & $\beta$ & OR & $\beta$ & OR \\
\hline Covid * & $0.27^{c}$ & 1.31 & $0.27^{c}$ & 1.31 & $0.39^{c}$ & 1.47 & $0.62^{\mathrm{b}}$ & 1.85 \\
\hline VS * -Non-IVS & - & - & - & - & - & - & $0.37^{\mathrm{b}}$ & 1.45 \\
\hline -IVS & - & - & - & - & - & - & $2.70^{c}$ & 14.90 \\
\hline \multicolumn{9}{|l|}{ Severity $\S$} \\
\hline Asymp. & $1.06^{c}$ & 2.90 & -0.06 & 0.94 & 1.01 & 2.76 & $-0.32^{c}$ & 0.74 \\
\hline Severe & $0.65^{c}$ & 1.91 & 1.58 & 4.84 & 1.84 & 6.34 & $0.45^{c}$ & 1.56 \\
\hline Heart dise.* & $0.23^{c}$ & 1.26 & $0.26^{c}$ & 1.30 & $0.3^{c}$ & 1.35 & $-0.03^{b}$ & 0.97 \\
\hline Lung dise. * & $0.24^{\mathrm{c}}$ & 1.27 & $0.28^{c}$ & 1.32 & $0.47^{c}$ & 1.61 & $0.08^{c}$ & 1.08 \\
\hline Diabetes * & 0.18 & 1.19 & $0.14^{c}$ & 1.15 & $0.3^{c}$ & 1.36 & $0.15^{c}$ & 1.16 \\
\hline Asthma * & $-0.16^{c}$ & 0.85 & $0.26^{c}$ & 1.29 & -0.04 & 0.96 & $-0.33^{c}$ & 0.72 \\
\hline Immunosu. * & $0.27^{c}$ & 1.31 & -0.02 & 0.98 & $0.28^{c}$ & 1.32 & $0.77^{c}$ & 2.17 \\
\hline Renal dise. * & $0.48^{c}$ & 1.62 & $-0.05^{a}$ & 0.95 & $0.32^{c}$ & 1.38 & $0.47^{c}$ & 1.60 \\
\hline Liver dise. * & $0.33^{c}$ & 1.40 & -0.06 & 0.94 & 0.38 & 1.46 & $0.68^{c}$ & 1.97 \\
\hline Obesity * & $0.48^{c}$ & 1.62 & $0.33^{c}$ & 1.38 & $0.68^{c}$ & 1.98 & $0.13^{c}$ & 1.14 \\
\hline Age & 0.01 & 1.01 & 0.01 & 1.01 & $0.02^{\mathrm{c}}$ & 1.02 & 0.04 & 1.05 \\
\hline Female $^{+}$ & -0.11 & 0.89 & $-0.06^{c}$ & 0.94 & $-0.18^{c}$ & 0.83 & -0.16 & 0.85 \\
\hline Race ${ }^{\mathbb{I}}$ - White & $0.08^{c}$ & 1.09 & 0.01 & 1.0 & $-0.11^{a}$ & 0.9 & -0.36 & 0.70 \\
\hline - Other & 0.001 & 1.0 & 0.001 & 1.0 & 0.05 & 1.06 & 0.06 & 1.06 \\
\hline
\end{tabular}

OR: Odds Ratio. $\beta$ : coefficient. P values a: $<0.05 ;$ b: $<0.01$; $:<0.001$. Ref. cat. ${ }^{*}$ : No; $\S$ : Mild; $\uparrow$ : Male; $\mathbb{I I}:$ Black.

In the Covid group there is a higher proportion of heart disease (33.4\% vs. $29.4 \%)$, diabetes $(25.2 \%$ vs. $18.9 \%)$, and obesity ( $4.7 \%$ vs. $22.6 \%)$; there is a lower proportion of lung disease (3.6\% vs. $7.4 \%)$, asthma (2.6\% vs. $5.7 \%)$, and immunosuppression (2.6\% vs. $4.5 \%$ ). Renal disease and liver disease are present in $4 \%$ and $1 \%$ of the cases in both groups. $51.6 \%$ of Covid cases and $49.7 \%$ of non-Covid cases have at least one comorbidity. 
Table 3 shows that Covid and severe cases have increased odds of worse outcomes (being admitted to ICU, needing non-IVS and IVS, and dying. The frequency of asymptomatic cases is very low, and this could be a reason for explaining the increased odds of worse outcomes in them.

Heart disease, lung disease, immunosuppression, renal disease, liver disease, diabetes, and obesity also have higher odds of worse outcomes. Patients suffering from asthma and females have lower odds of bad outcomes. With the increase of age, the odds of bad outcomes increase. There are no differences when considering race, however, it is worth noting that the odds of dying are $30 \%$ lower in whites compared to blacks. Poisson regression showed that there is a significant increase in the length of hospitalization and ICU stay of $30 \%$ and $40 \%$ in Covid patients and a decrease by $10 \%$ and $7 \%$ in asthma patients; the effects of other variables were not clinically significant. The analysis suggests that asthma plays a protective role, which research shows to be biologically plausible [12].

\section{Discussion}

This is an observational study utilizing data generated from surveillance which are subject to errors. E.g. the record sheet requires reporting of chronic diseases, but there are observations having congenital heart disease being reported as having chronic heart disease; this may explain some of the reported effect sizes. Missing data is another issue present in the dataset.

\section{Conclusion}

Hospitalized SARI cases analysis is important in exploring the factors influencing outcomes.

Author Contributions: Karl E. Peace contributed to the conceptualization, methodology, supervision, project administration, writing-review and editing. Mario Keko contributed to data curation, investigation, software, validation, formal analysis, visualization, and writing - original draft preparation.

Funding: This research received no external funding.

Acknowledgments: The authors would like to thank Aurela Nikaj for proofreading the draft of the manuscript.

Conflicts of Interest: The authors declare no conflict of interest.

\section{Appendix}

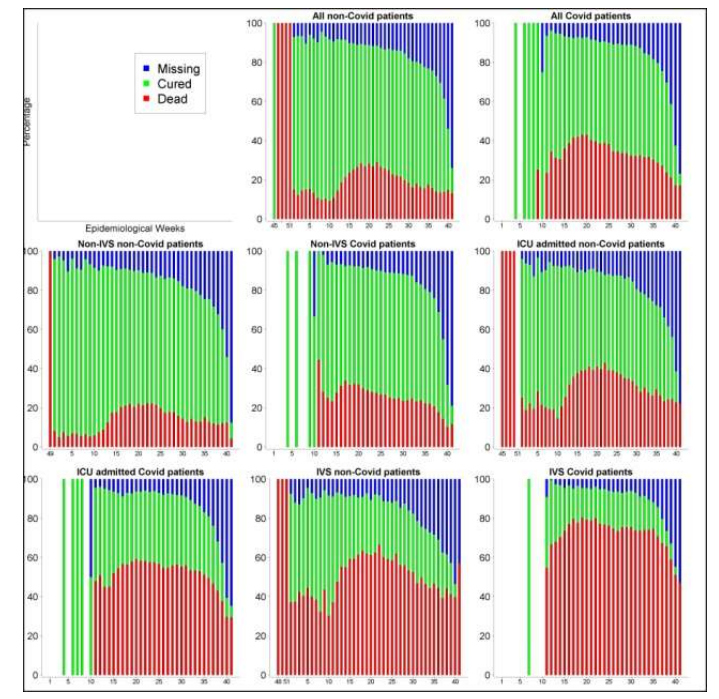

(a)

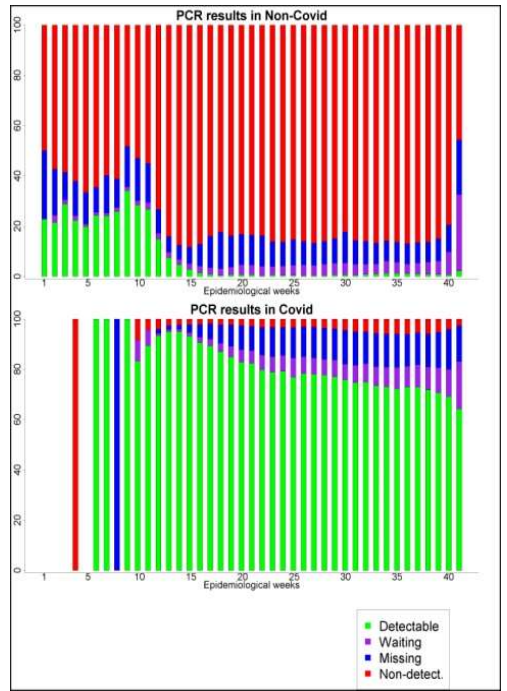

(b) 
Figure A1. (a) Duration of hospitalization and ICU stay by epidemiological week and groups (b) PCR test results by epidemiological week and groups.

Table 1. Severity determination.

\begin{tabular}{ccc}
\hline None of below & At least one of below & Severity \\
\hline $\mathrm{a}, \mathrm{b}, \mathrm{c}, \mathrm{d}, \mathrm{e}, \mathrm{f}, \mathrm{g}, \mathrm{h}, \mathrm{i}$ & - & Asymptomatic \\
\hline $\mathrm{f}, \mathrm{g}, \mathrm{h}, \mathrm{i}$ & $\mathrm{a}, \mathrm{b}, \mathrm{c}, \mathrm{d}, \mathrm{e}$ & Mild \\
\hline & $\mathrm{f}, \mathrm{g}, \mathrm{h}, \mathrm{i}$ & Severe \\
\hline
\end{tabular}

a: Fever; b: Cough; c: Throat pain; d: Diarrhea; e: Vomitting; f: Dyspnea; g: Resp. discomfort.; h: ${ }_{2}$ Sat. $<95 \%$; i: Abnormal chest X-Ray.

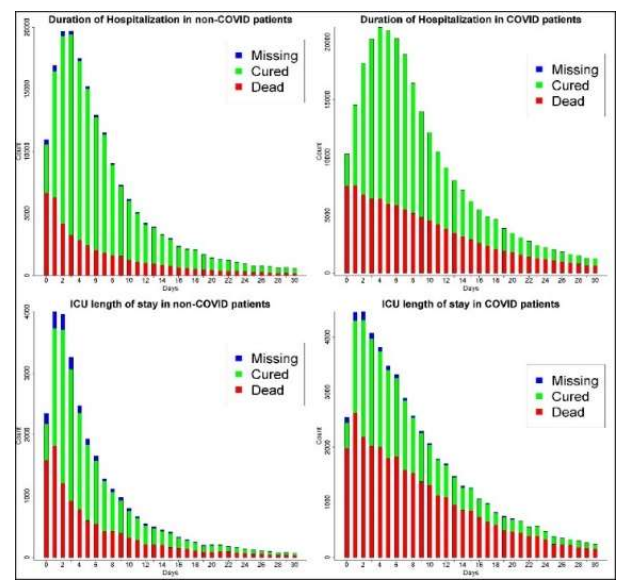

Figure A1. Hospitalization and ICU stay length.

\section{References}

1. Gorbalenya, A.E., et al., The species Severe acute respiratory syndrome-related coronavirus: classifying 2019-nCoV and naming it SARSCoV-2. Nature Microbiology, 2020. 5(4): p. 536-544.

2. Dhama, K., et al., Coronavirus Disease 2019-Covid-19. Clin Microbiol Rev, 2020. 33(4).

3. Li, N., et al., Molecular diagnosis of Covid-19: Current situation and trend in China (Review). Exp Ther Med, 2020. $20(5)$ : p. 13.

4. Guglielmi, G., The explosion of new coronavirus tests that could help to end the pandemic. Nature, 2020. 583(7817): p. 506-509.

5. Hallal, P.C., et al., SARS-CoV-2 antibody prevalence in Brazil: results from two successive nationwide serological household surveys. Lancet Glob Health, 2020. 8(11): p. e1390-e1398.

6. Plataforma IVIS. Available from: http://plataforma.saude.gov.br/coronavirus/dados-abertos/ (accessed on 12 November 2020).

7. FICHA DE REGISTRO INDIVIDUAL - CASOS DE SÍNDROME RESPIRATÓRIA AGUDA GRAVE HOSPITALIZADO. Available from: http://plataforma.saude.gov.br/coronavirus/dados-abertos/sivep-gripe/ficha-SIVEP-GRIPE-SRAG-hospital-Sivepgripe.pdf (accessed on 12 November 2020).

8. Clinical Spectrum of SARS-CoV-2 Infection. Available from: https://www.covid19treatmentguidelines.nih.gov/overview/clinical-spectrum/ (accessed on 14 November 2020).

9. $\quad$ Agresti, A., Logistic Regression, in An Introduction to Categorical Data Analysis. 2007, Wiley. p. 106-109.

10. Agresti, A., Generalized Linear Models for Count Data, in An introduction to categorical data analysis. 2007, Wiley. p. 74-83.

11. de Souza, W.M., et al., Epidemiological and clinical characteristics of the Covid-19 epidemic in Brazil. Nature Human Behaviour, 2020. 4(8): p. 856-865.

12. Carli, G., et al., Is asthma protective against Covid-19? Allergy, 2020: p. 10.1111/all.14426. 